\title{
Female survivors of intimate partner violence and risk of depression, anxiety and serious mental illness
}

Joht Singh Chandan, Tom Thomas, Caroline Bradbury-Jones, Rebecca Russell, Siddhartha Bandyopadhyay* , Krishnarajah Nirantharakumar* and Julie Taylor*

\section{Background}

Internationally, intimate partner violence (IPV) cohorts have demonstrated associations with depression and anxiety. However, this association has not yet been described in a UK population, nor has the association with serious mental illness (SMI).

\section{Aims}

To explore the relationship between IPV exposure and mental illness in a UK population.

\section{Method}

We designed a retrospective cohort study whereby we matched 18547 women exposed to IPV to 74188 unexposed women. Outcomes of interest (anxiety, depression and SMI) were identified through clinical codes

\section{Results}

At baseline, 9174 (49.5\%) women in the exposed group had some form of mental illness compared with 17768 (24.0\%) in the unexposed group, described as an adjusted odds ratio of 2.62 (95\% $\mathrm{Cl} 2.52-2.72)$. Excluding those with mental illness at baseline, 1254 exposed women (incidence rate 46.62 per 1000 person-years) went on to present with any type of mental illness compared with 3119 unexposed women (incidence rate 14.93 per 1000 person-years), with an alRR of 2.77 (95\% Cl 2.58-2.97) Anxiety (aIRR 1.99, 95\% Cl 1.80-2.20), depression (aIRR 3.05, 95\% $\mathrm{Cl} 2.81-3.31$ ) and SMI (aIRR 3.08, 95\% Cl 2.19-4.32) were all associated with exposure to IPV

\section{Conclusions}

IPV remains a significant public health issue in the UK. We have demonstrated the significant recorded mental health burden associated with IPV in primary care, at both baseline and following exposure. Clinicians must be aware of this association to reduce mental illness diagnostic delay and improve management of psychological outcomes in this group of patients.

\section{Declaration of interest}

None.

\section{Keywords}

Outcome studies; depressive disorders; epidemiology; anxiety disorders; trauma.

\section{Copyright and usage}

(c) The Royal College of Psychiatrists 2019
Intimate partner violence (IPV), seen as a violation of human rights, remains a prevalent global public health issue affecting as many as one in three women. ${ }^{1,2}$ Being a survivor of IPV is associated with a wide range of poor health outcomes. ${ }^{3,4}$ For example, survivors of IPV undertake more harmful lifestyle choices such as smoking ${ }^{5}$ and excessive alcohol use. ${ }^{6}$ There is a strong relationship between being a survivor of IPV and poor mental health outcomes. The pathway that leads to this association is complex, with there likely being biochemical, psychological and environmental risk factors that predispose survivors of abuse to go on to develop poor mental health outcomes. ${ }^{7}$ Previous systematic reviews of observational studies have identified associations between being a survivor of IPV with depression, suicide, post-traumatic stress disorder and prenatal depressive symptoms. ${ }^{8-12}$ In a recent review of all cohorts exploring the impact of IPV on female survivors' physical and mental health, ${ }^{4} 13$ of these studies explored the relationship between IPV and depression. None of these 13 studies were set in the UK. Although there are challenges in discerning the temporality of this relationship, this association appeared to be bidirectional. When depression was explored as both a dependent and independent variable, a positive correlation remained. Only one cohort study set in the USA ascertained a positive relationship between IPV and subsequent diagnosis of generalised anxiety disorder. ${ }^{13}$ We identified no cohort studies that investigated the relationship between IPV and subsequent diagnosis of serious mental illness (SMI), including schizophrenia, bipolar disorder, mania and other forms

* Equal contributors. of non-affective psychoses. An alternative review ${ }^{12}$ compiling case-control and cross-sectional data highlighted just one study investigating the relationship between IPV and bipolar disorder, which appear to be linked. ${ }^{14} \mathrm{~A}$ separate more recent review ${ }^{15}$ exploring the prevalence of experiencing domestic violence within the past year in groups of patients with severe mental illness, reported a prevalence of domestic violence exposure between 15 and $22 \%$.

Because of the prevalence of IPV, there appears to be an association between mental health burden and exposure to IPV. So far there has not been a UK-based cohort study assessing the relationship between IPV and anxiety, depression and SMI. As well as shedding new light on the relationship between IPV and anxiety/SMI, it is also important to quantify the extent of this burden to enable planning of targeted mental health services in the UK for this group at risk. Therefore, we aimed to explore this association by using primary care records derived from The Health Improvement Network (THIN) database.

\section{Method}

\section{Study design and data source}

This study was a population-based, retrospective, open cohort study using the THIN database, comparing female patients coded with previous exposure of IPV with female patients not coded to have experienced IPV. The THIN database consists of UK electronic medical records derived from over 750 general practices (family 
practices), comprising approximately 3.6 million patients at the time of this study. THIN is deemed demographically representative of the UK population. ${ }^{16}$ Information regarding patients' symptoms and diagnoses are recorded using the Read code hierarchy system. ${ }^{17,18}$ To reduce under-recording of events, general practices were included 12 months following their instalment of electronic practice records or from the practice's acceptable mortality recording date.

\section{Study population}

The study period was set between 1 January 1995 and 1 December 2017. During this period, women over the age of 18 years who had documented exposure to IPV, noted through Read codes by their General Practitioner (GP), were deemed to be our exposed group. The index date for individuals in the exposed group was taken to be the first inserted Read code relating to IPV exposure once a patient was eligible to take part in the study or, alternatively, the study start date for patients with a previous record of IPV (prevalent cases). To mitigate immortality time bias, ${ }^{19}$ the same index date was assigned to the corresponding unexposed patient. Each exposed survivor of IPV was matched with up to four control patients, who had not been documented to have a Read code relating to IPV exposure, from general practices within the database forming the unexposed group. Controls from the unexposed group were matched individually to cases based on age at index date ( \pm 1 year), and gender.

The primary outcome explored in this study was the development of mental illness, which comprised depression, anxiety, SMI and a combination of these three. If individuals in either group had a diagnosis of one of the primary outcome diagnoses before the study start date, they were excluded. However, as much of the exposed population experienced mental illness at baseline, we have also described the risk of mental illness at entry to the study.

Covariates that affect the development of mental illness were included in the baseline data for this population. These included

\begin{tabular}{|c|c|c|}
\hline $\begin{array}{l}\text { Baseline characteristics } \\
\text { (s.d. or \%) }\end{array}$ & Exposed group & Unexposed group \\
\hline Number of patients & 18547 & 74188 \\
\hline Follow-up period (person-years) & 2.2 (s.d. 2.3) & 3.2 (s.d. 2.8) \\
\hline Age (years) & 36.9 (s.d. 12.5) & 36.9 (s.d. 12.5) \\
\hline \multicolumn{3}{|l|}{ Body mass index } \\
\hline$<25 \mathrm{~kg} / \mathrm{m}^{2}$ & 7916 (42.7\%) & $32330(43.6 \%)$ \\
\hline $25-30 \mathrm{~kg} / \mathrm{m}^{2}$ & 3999 (21.6\%) & $16346(22.0 \%)$ \\
\hline$>30 \mathrm{~kg} / \mathrm{m}^{2}$ & $3568(19.2 \%)$ & $13934(18.8 \%)$ \\
\hline Not available & $3064(16.5 \%)$ & $11578(15.6 \%)$ \\
\hline Current smoking & $8096(44.7 \%)$ & $16039(21.6 \%)$ \\
\hline \multicolumn{3}{|l|}{ Drinking status } \\
\hline Non-drinker & $5149(27.8 \%)$ & $13771(18.6 \%)$ \\
\hline Drinker not excess & $8353(45.0 \%)$ & $44112(59.5 \%)$ \\
\hline Excessive drinker & $1870(10.1 \%)$ & 1580 (2.1\%) \\
\hline Not available & 3175 (17.1\%) & 14725 (19.9\%) \\
\hline \multicolumn{3}{|l|}{ Townsend Deprivation Index score } \\
\hline (Least deprived) 1 & $1773(10.1 \%)$ & $14160(20.2 \%)$ \\
\hline 2 & $2104(12.0 \%)$ & $12881(18.4 \%)$ \\
\hline 3 & 3149 (17.9\%) & 13548 (19.3\%) \\
\hline 4 & 4215 (24.0\%) & 12601 (18.0\%) \\
\hline 5 & $4266(24.3 \%)$ & 9330 (13.3\%) \\
\hline Not available & $2068(11.8 \%)$ & $7691(11.0 \%)$ \\
\hline \multicolumn{3}{|l|}{ Mental illness at baseline } \\
\hline Depression & $7533(40.6 \%)$ & $13247(17.9 \%)$ \\
\hline Anxiety & $3734(20.1 \%)$ & $7586(10.2 \%)$ \\
\hline Serious mental illness (SMI) & $478(2.6 \%)$ & $607(0.8 \%)$ \\
\hline $\begin{array}{l}\text { Combined depression, anxiety } \\
\text { and SMI }\end{array}$ & $9174(49.5 \%)$ & $17768(24.0 \%)$ \\
\hline
\end{tabular}

body mass index, deprivation assessed by the Townsend Deprivation Index score, ${ }^{20}$ smoking status and alcohol use.

Read code lists for the exposure and outcomes are provided in the supplementary material (Supplementary File 1 available at https://doi.org/10.1192/bjp.2019.124).

\section{Statistical analysis}

Categorical baseline data were described by proportions. Continuous data were described by means and s.d. Missing data is highlighted in Table 1. As much of the exposed population had mental illness at baseline, we have also described the odds of having mental illness at baseline between the exposed and unexposed groups. This has been described by a logistic regression, which provides an unadjusted odds ratio and adjusted odds ratio (aOR), factoring in the covariates of interest above. Odds ratios were calculated with 95\% CIs and statistical significance was set at $P<0.05$. Where there was missing data in our covariates, they were treated as a separate missing category and included in the regression analysis.

After patients with the mental illness of interest at baseline were excluded, Poisson regression was used to calculate an incidence rate ratio (IRR) for each outcome of interest during the study period. Following adjustment for important documented covariates that may independently affect the outcome of interest, we have calculated and presented an adjusted incidence rate ratio (aIRR). IRRs were calculated with $95 \%$ CIs and statistical significance was set at $P<0.05$. These are presented for each of the outcomes of interest: depression, anxiety, SMI and a composite of the three.

To account for survival bias, a sensitivity analysis was conducted, excluding prevalent cases of IPV and thereby including only incident cases of IPV with their respective controls. To account for possible misclassification of mental illness coding, we have conducted a second sensitivity analysis whereby we have explored the incidence of a new starting prescription of anxiolytic, antidepressant and antipsychotic medications (drug codes taken from relevant British National Formulary (https://bnf.nice.org.uk/) chapters are presented in Supplementary File 1), for the main results. Stata version 14.2 for MacOS software was used to conduct all analysis throughout the study.

\section{Ethical approval and data accessibility}

Anonymised data was used throughout the study, provided by the data provider IQVIA to University of Birmingham. Studies using the THIN database have had initial ethical approval from the National Health Service South-East Multicentre Research Ethics Committee, subject to prior independent scientific review. The Scientific Review Committee (IMS Health) approved the study protocol (reference number SRC18THIN034) before its undertaking. The full data-set and statistical analysis code are available from author K.N. (k.nirantharan@bham.ac.uk).

\section{Results}

\section{Baseline characteristics}

A total of 18547 women who had been documented to have experienced IPV were matched to 74188 controls by age and gender. The mean length of follow-up in the exposed group was shorter compared with the unexposed group. Mean age in both groups was similar. Obesity, prevalence of smoking and number of women who were excessively drinking at baseline were significantly higher in the exposed group compared with the unexposed. The exposed group were also more socioeconomically deprived at baseline. At baseline, a high proportion of individuals who were exposed to IPV compared with the unexposed group had experienced mental 
illness in the form of depression (40.6\%), anxiety (20.1\%) or SMI $(2.6 \%)$ compared with the unexposed group at $17.9 \%, 10.2 \%$ and $0.8 \%$, respectively. Characteristics of both populations are described in detail in Table 1.

\section{The odds of having depression, anxiety and SMI at baseline}

This odds of having mental illness in the population being studied is described in Table 2 and demonstrated in Fig. 1. As described above, at baseline there was a significantly higher burden of mental illness. At study start date, $49.5 \%(n=9174 / 18457)$ of the exposed group had experienced any type of mental health outcome compared with $24.0 \%$ ( $n=17768 / 74188)$ of the unexposed group. This translated to an increased odds ratio of 3.11 (95\% CI 3.01-3.21). Following adjustment for covariates, this translated to an aOR of 2.62 (95\% CI 2.52-2.72). When subcategorised by outcomes, the exposed group experienced a higher risk of having depression, anxiety and SMI following adjustment, at the study start date compared with the unexposed group. The aORs were 2.61 (95\% CI 2.512.71), 1.91 (95\% CI 1.82-2.01) and 2.13 (95\% CI 1.86-2.43), respectively.

\section{Association between IPV and depression, anxiety and SMI}

The main results are presented in Table 3 and Fig. 1. During our study period 1254 patients (incidence rate 46.62 per 1000 personyears) presented with any type of mental illness in the exposed group compared with 3119 in the unexposed group (incidence rate 14.93 per 1000 person-years). This translated to a significant unadjusted increased IRR of 3.12 (95\% CI 2.92-3.33). Following adjustment, this remained significant (aIRR 2.77, 95\% CI 2.582.97). Anxiety (aIRR 1.99, 95\% CI 1.80-2.20), depression (aIRR 3.05, 95\% CI 2.81-3.31) and SMI (aIRR 3.08, 95\% CI 2.19-4.32) were all positively associated following exposure to IPV.

\section{Sensitivity analysis}

Our sensitivity analysis, consisting of incident-only cases during the study period (Table 4), also remained congruent with the main results. The baseline characteristics of this group (Supplementary File 2) were similar in nature to the main analysis, with similar differences presenting in average follow-up time, body mass index, smoking, deprivation and alcohol use at baseline. All mental illness in the sensitivity analysis remained strongly associated, with an incidence ratio of 47.29 per 1000 person-years in the exposed group compared with 14.57 per 1000 person-years in the unexposed group, resulting in an aIRR of 2.89 (95\% CI 2.623.18). Similarly, anxiety (aIRR $2.11,95 \%$ CI $1.84-2.41$ ), depression (aIRR 3.09, 95\% CI 2.76-3.46) and SMI (aIRR 3.06 95\% CI 1.855.07) remained strongly associated with exposure to IPV. In addition, the odds of mental illness at baseline was significantly higher in the exposed group in this sensitivity analysis.

The results of our second sensitivity analysis (Supplementary File 3) also support the main findings. At baseline we noted similarly increased odds of having a prescription indicative of mental illness (aOR 3.20, 95\% CI 3.08-3.32). When subcategorised by prescription types the aOR for anxiolytics, antidepressants and antipsychotic medication were 2.52 (95\% CI 2.36-2.70), 3.25 (95\% CI 3.133.38 ) and 1.95 (95\% CI 1.82-2.10), respectively. When exploring the association between a new prescription of an agent used for treating mental illness, we also noted a similarly positive effect size (aIRR 2.37, 95\% CI 2.24-2.50). This remained positive for each subtype of prescription: anxiolytics (aIRR 1.67, 95\% CI 1.55-
1.80), antidepressants (aIRR 2.58, 95\% CI 2.44-2.73) and antipsychotics (aIRR 1.64, 95\% CI 1.52-1.77).

\section{Discussion}

\section{Summary of key results}

In summary, the results suggest a strong association between exposure to IPV and incident mental illness (aIRR 2.77, 95\% CI 2.582.97 ) in this UK primary care data-set. This relationship was significant when assessing the incidence of anxiety, depression and SMI. These relationships remained positive following sensitivity analysis considering only incident cases as well as prescriptions for treatment of mental illness. Another key finding was that the odds of having mental illness at baseline in the IPV group was significantly higher than the unexposed group (aOR 2.62, 95\% CI 2.52-2.72). This suggests that there is a higher likelihood of having mental illness before recorded exposure of IPV, but also for those who become exposed, their risk of mental illness continues to increase.

\section{Relationship to current literature}

To our knowledge, this is the first cohort study assessing recorded incident depression, anxiety and SMI following exposure to recorded IPV within the UK, using primary care records. Therefore, it is difficult to make comparisons relating to the expected incidence of these outcomes in a UK population. However, this study is consistent with previous work undertaken globally, which suggests a relationship between exposure to IPV and subsequent mental illness. It was noted in a recent meta-analysis, ${ }^{4}$ when depression is considered as a dependent variable as in this case, the pooled odds ratio from previous cohort studies was 1.76 (95\% CI 1.26-2.44). Our result is similarly positively associated (aIRR 3.05, 95\% CI 2.81-3.31).

Comparatively, our study (aIRR 1.99, 95\% CI 1.80-2.20) also supports a link associating anxiety and IPV exposure identified in another cohort study. ${ }^{13}$ However, the main aim of that cohort study was not to ascertain the relationship specifically of IPV exposure to anxiety, but to assess the relationship of multiple factors relating to housing conditions and the development of mental illness, whereas in our study, the development of anxiety following IPV exposure was a primary outcome measure. Aside from cohort studies, there have been several other observational studies (casecontrol and cohort) $)^{12}$ that have identified a positive association between IPV and anxiety (pooled odds ratio, 4.06; 95\% CI 2.396.97). Our study supports this association in a UK population.

Previously, there has been limited research exploring the relationship between SMI and IPV; however, of the work that has been done, ${ }^{12,14,15}$ there has been a strong positive association, which our study affirms. The results of our study clearly show a strong association between the development of poor mental health outcomes following IPV in a UK setting, which are of importance in psychiatric and primary care settings. It has been shown in a variety of studies that survivors of IPV experience significant barriers $^{21-24}$ in receiving the necessary healthcare support they often require. One of the key barriers relates to the identification of IPV exposure in women who present to healthcare services. It is clear from this study that there is still significant under-recording of IPV in this database, considering estimates of the prevalence of IPV could be as high as one in three women. ${ }^{2}$ However, there still remains a strong association with poor mental health outcomes, which does suggest that when women present with depression, anxiety and SMI, a past history of IPV should be explored to aid in management plans. Our study has also shown the increased prevalence of mental illness at baseline in the IPV cohort, suggesting 


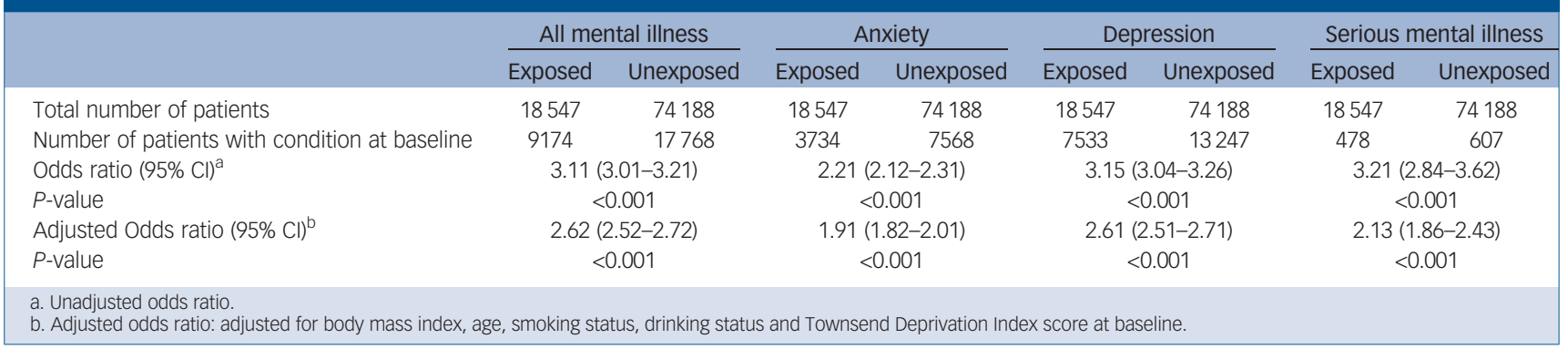

that protective mental health interventions should be introduced early in their treatment plan.

The findings of this study are timely in relation to changes over the past 5 years in current practice within the UK and globally, relevant to both the enquiry of IPV and referral to supportive interventions. Previous literature had highlighted failures of UK mental health services in the identification of exposure to IPV in patients utilising their services, and in addition to poor integration of these services with appropriate referral pathways for these survivors. ${ }^{25}$ In response to evidence suggesting the negative effects of such a model, the National Institute for Health and Care Excellence introduced the PH50 guidance in 2014, which highlighted the importance for multi-agency staff to enquire routinely about domestic violence and abuse and provide supportive options for referral. ${ }^{26}$ Within the same year, the World Psychiatric Association (WPA) isolated the importance of identifying gender-based domestic violence in psychiatric consultations, and this has been highlighted within the WPA curriculum for trained mental health professionals. ${ }^{27,28}$ The result of these changes is hopefully leading to a UK clinical environment that identifies the needs of possible survivors of IPV who may have otherwise been missed. We have since seen the introduction of the
Identification and Referral to Improve Safety project) in several sites across the $\mathrm{UK},{ }^{23}$ which aims to train and educate GPs in the enquiry of abuse and care pathways of survivors. In addition, another recent project funded by the UK Government is Pathfinder, a 3-year project started in 2017 aiming to establish comprehensive health practice in relation to domestic abuse, to also bridge gaps in provision for the cohort of survivors who may otherwise slip through the net because of lack of identification in clinical settings. ${ }^{29}$ Although our study does identify that there is perhaps a significant burden of unmet need in a subgroup of women who have experienced IPV, hopefully within the UK we are beginning to see changes within current practice. However, there is still a need to ensure that we are not missing potential opportunities to aid survivors in the disclosure of IPV, and referral to supportive services.

\section{Study limitations}

The use of this data-set relies upon the accuracy of imputation of Read codes by GPs. In this study, we were unable to validate the Read codes of IPV and mental illness with participating practices. This is an important future area of research that will help improve the validity of results in future work. However, we were able to conduct a sensitivity analysis using prescriptions relating

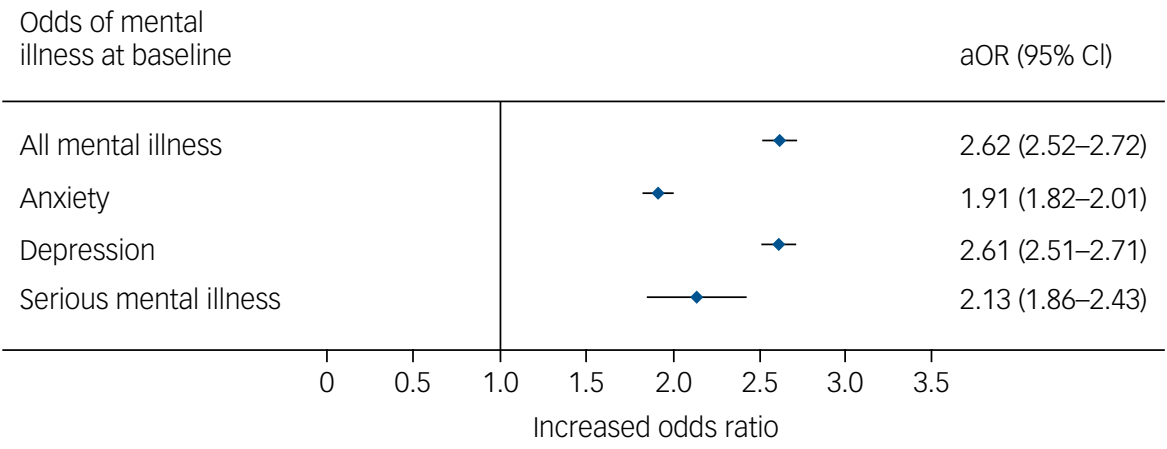

Risk of mental illness following exposure to IPV

$\operatorname{alRR}(95 \% \mathrm{Cl})$

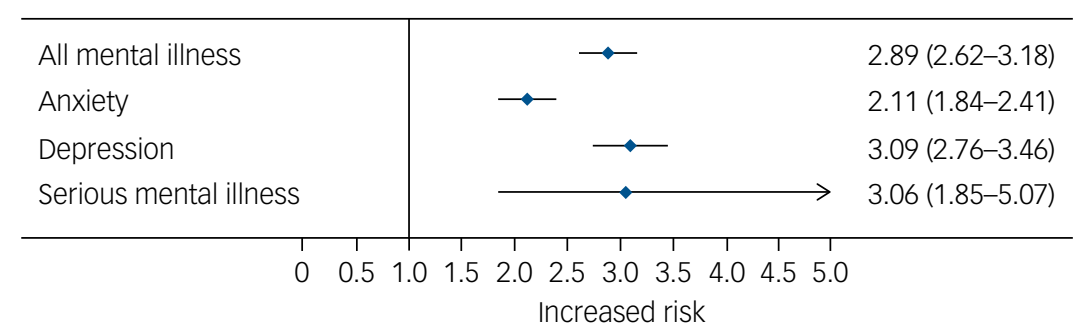


Table 3 Risk of mental illness development between the exposed and unexposed groups

\begin{tabular}{|c|c|c|c|c|c|c|c|c|}
\hline & \multicolumn{2}{|c|}{ All mental illness } & \multicolumn{2}{|c|}{ Anxiety } & \multicolumn{2}{|c|}{ Depression } & \multicolumn{2}{|c|}{ Serious mental illness } \\
\hline & Exposed & Unexposed & Exposed & Unexposed & Exposed & Unexposed & Exposed & Unexposed \\
\hline Number of patients & 9373 & 56420 & 14813 & 66602 & 11014 & 60941 & 18069 & 73581 \\
\hline Numbers of outcomes & 1254 & 3119 & 633 & 1585 & 1020 & 2114 & 69 & 93 \\
\hline Person-years & 26899 & 208940 & 46869 & 254551 & 32542 & 229400 & 59061 & 268735 \\
\hline Incidence rate (per 1000 person-years) & 46.62 & 14.93 & 13.50 & 6.23 & 31.34 & 9.22 & 1.17 & 0.32 \\
\hline Incidence rate ratio $(95 \% \mathrm{Cl})^{\mathrm{a}}$ & \multicolumn{2}{|c|}{$3.12(2.92-3.33)$} & \multicolumn{2}{|c|}{$2.19(1.98-2.38)$} & \multicolumn{2}{|c|}{$3.40(3.16-3.67)$} & \multicolumn{2}{|c|}{$3.60(2.63-4.92)$} \\
\hline$P$-value & \multicolumn{2}{|c|}{$<0.001$} & \multicolumn{2}{|c|}{$<0.001$} & \multicolumn{2}{|c|}{$<0.001$} & \multirow{2}{*}{\multicolumn{2}{|c|}{$\begin{array}{c}<0.001 \\
3.08(2.19-4.32)\end{array}$}} \\
\hline Adjusted incidence rate ratio $(95 \% \mathrm{Cl})^{b}$ & \multirow{2}{*}{\multicolumn{2}{|c|}{$2.77(2.58-2.97)$}} & \multirow{2}{*}{\multicolumn{2}{|c|}{$1.99(1.80-2.20)$}} & \multicolumn{2}{|c|}{$3.05(2.81-3.31)$} & & \\
\hline$P$-value & & $<0.001$ & & & \multicolumn{2}{|c|}{$<0.001$} & \multicolumn{2}{|c|}{$<0.001$} \\
\hline
\end{tabular}

\begin{tabular}{|c|c|c|c|c|c|c|c|c|}
\hline & \multicolumn{2}{|c|}{ All mental illness } & \multicolumn{2}{|c|}{ Anxiety } & \multicolumn{2}{|c|}{ Depression } & \multicolumn{2}{|c|}{ Serious mental illness } \\
\hline & Exposed & Unexposed & Exposed & Unexposed & Exposed & Unexposed & Exposed & Unexposed \\
\hline Number of patients & 4506 & 27086 & 7067 & 32023 & 5269 & 29217 & 8708 & 35343 \\
\hline Numbers of outcomes & 668 & 1547 & 345 & 793 & 531 & 1044 & 30 & 42 \\
\hline Person-years & 14126 & 106213 & 24724 & 129622 & 17186 & 116564 & 31694 & 146166 \\
\hline Incidence rate (per 1000 person-years) & 47.29 & 14.57 & 13.95 & 6.12 & 30.90 & 8.96 & 0.95 & 0.29 \\
\hline Incidence rate ratio $(95 \% \mathrm{Cl})^{\mathrm{a}}$ & \multicolumn{2}{|c|}{$3.25(2.97-3.56)$} & \multicolumn{2}{|c|}{$2.28(2.01-2.59)$} & \multicolumn{2}{|c|}{$3.45(3.11-3.83)$} & \multicolumn{2}{|c|}{$3.29(2.06-5.36)$} \\
\hline$P$-value & \multirow{2}{*}{\multicolumn{2}{|c|}{$2.89(2.62-3.18)$}} & \multicolumn{2}{|c|}{$<0.001$} & \multicolumn{2}{|c|}{$<0.001$} & \multirow{2}{*}{\multicolumn{2}{|c|}{$\begin{array}{c}<0.001 \\
306(185-507)\end{array}$}} \\
\hline Adjusted incidence rate ratio $(95 \% \mathrm{Cl})^{\mathrm{a}}$ & & & & & & & & \\
\hline$P$-value & \multicolumn{2}{|c|}{$<0.001$} & \multicolumn{2}{|c|}{$<0.001$} & \multicolumn{2}{|c|}{$<0.001$} & \multicolumn{2}{|c|}{$<0.001$} \\
\hline
\end{tabular}

to mental illness and this show congruent results. An important limitation in this study, is the number of women identified as exposed to IPV appears extremely low compared with previous UK and global estimates of IPV., ${ }^{2,30}$ Using data derived from the total population during the final year of the study period, we have identified the point prevalence of exposure to IPV in women to be $0.5 \%$, which is low. This highlights another important message of this study, which is to bring to light the need for improved recording of IPV in primary care. Thus, it is possible that members of the unexposed group may actually have experienced IPV but were misclassified, possibly underestimating our effect size. Alternatively, we may have only identified women with severe IPV who chose to present to their GP, overestimating our effect size. In relation to this, because of very low recorded numbers, we were unable to conduct a subgroup analysis of physical, sexual or emotional abuse in the IPV cohort. Therefore, in future work, if coding is improved it will be important to tease out this relationship further. An interesting point of note is that following age and gender matching, there is a shorter follow-up period in the exposed group, which may be representative of the extent of geographical moves women who experience IPV may be making following disclosure of abuse. One of the challenges in this study design is accounting for reverse causality. As discussed in the literature, ${ }^{4}$ there also appears to be a reverse relationship where individuals with mental illness appear to be more likely to become victims of IPV. We attempted to account for this by excluding all survivors with a pre-existing mental health diagnosis. Because of diagnostic delay in identifying mental illnesses, ${ }^{31}$ it is likely that some individuals will have begun to experience symptoms of these conditions before their index date in the study. One fact to note is that at baseline the exposed group had considerably higher odds of having mental illness at baseline (aOR 2.78, 95\% CI 2.68-2.89). This could perhaps be owing to a significant delay in presentation of IPV to their GP, meaning that mental illness may precede the recorded exposure to IPV.
In summary, we have undertaken the first cohort study in the UK to use primary care data to ascertain the relationship between recorded IPV and mental illness. In light of the study's limitations, particularly relating to under recording of IPV, we still found an association between IPV exposure, with a twofold increase in the risk of developing anxiety, and a threefold risk increase of developing depression and SMI. Because of the sizeable public health burden posed by IPV, which is mostly under-reported, physicians should continue to pay particular attention to identifying individuals in this group. Early identification of such exposure in women presenting with depression, anxiety and SMI may improve psychological outcomes if a targeted management therapy is used. Further work is needed to explore the dose-dependent relationship between abuse and poor mental health, as well as a greater understanding of the pathway behind this. Also, this question should be explored in other UK cohorts to identify the extent of under-recording and test the effect size we have noted.

Joht Singh Chandan (D, MBBS, MFPH, BSC, Academic Clinical Fellow in Public Health, Institute of Applied Health Research, College of Medical and Dental Sciences, University of Birmingham, UK; Tom Thomas, MBBS, Clinical Research Fellow, Institute of Applied of Birmingham, UK; Tom Thomas, MBBS, Clinical Research Fellow, Institute of Applied
Health Research, College of Medical and Dental Sciences, University of Birmingham, UK; Caroline Bradbury-Jones, PhD, MA, RN, Reader in Nursing, School of Nursing, College of Medical and Dental Sciences, University of Birmingham, UK; Rebecca Russell, MBChB, MA (Cantab), MPH, DFPH, Public Health Registrar, Institute of Applied Health Research, College of Medical and Dental Sciences, University of Birmingham, UK; Siddhartha Bandyopadhyay, PhD, MA, BSC, Professor of Economics and Director of the Centre of Crime, Justice and Policing, Department of Economics, University of Birmingham, UK; Krishnarajah Nirantharakumar, MD, MBBS, MFPH, PGDip, MPH, MRCP, Senior Clinical Lecturer, Institute of Applied Health Research, College of Medical and Dental Sciences, University of Birmingham, UK; Julie Taylor, PhD, RN, MSC, BSC (Hons), RNT, FRCN, Professor of Child Protection, School of Nursing, College of Medical and Dental Sciences, University of Birmingham, Birmingham Women's and Children's Hospitals NHS Foundation Trust, UK

Correspondence: Julie Taylor, School of Nursing, College of Medical and Dental Sciences, Vincent Drive, University of Birmingham B152TT, UK. Email: j.taylor.1@bham.ac.uk First received 27 Nov 2018, final revision 23 Apr 2019, accepted 26 Apr 2019 


\section{Supplementary material}

Supplementary material is available online at https://doi.org/10.1192/bjp.2019.124.

\section{References}

1 Garcia-Moreno C, Jansen HAFM, Ellsberg M, Heise L, Watts $\mathrm{CH}$; WHO Multicountry Study on Women's Health and Domestic Violence against Women Study Team. Prevalence of intimate partner violence: findings from the WHO multi-country study on women's health and domestic violence. Lancet 2006; 368: 1260-9.

2 World Health Organization (WHO). Violence against Women. WHO, 2018 (http:// www.who.int/mediacentre/factsheets/fs239/en/).

3 Black MC, Basile KC, Breidling MJ, Smith SG, Walters ML, Merrick MT et al. National Intimate Partner and Sexual Violence Survey 2010 Summary Report. Centers for Disease Control and Prevention, 2010 (https://www.cdc.gov/ ViolencePrevention/pdf/NISVS_Report2010-a.pdf).

4 Bacchus L, Ranganathan M, Watts C, Devries K. Recent intimate partner violence against women and health: a systematic review and meta-analysis of cohort studies. BMJ Open 2018; 8: e019995.

5 Crane $\mathrm{CA}$, Hawes SW, Weinberger $\mathrm{AH}$. Intimate partner violence victimization and cigarette smoking. Trauma Violence Abus 2013; 14: 305-15.

6 La Flair LN, Bradshaw CP, Storr CL, Green KM, Alvanzo AAH, Crum RM. Intimate partner violence and patterns of alcohol abuse and dependence criteria among women: a latent class analysis. J Stud Alcohol Drugs 2012; 73: 351-60.

7 Delara M. Mental health consequences and risk factors of physical intimate partner violence. Ment Health Fam Med 2016; 12: 119-25.

8 Reingle Gonzalez JM, Jetelina KK, Olague S, Wondrack JG. Violence against women increases cancer diagnoses: results from a meta-analytic review. Prev Med (Baltim) 2018; 114: 168-79.

9 Devries KM, Mak JY, Bacchus LJ, Child JC, Falder G, Petzold M, et al. Intimate partner violence and incident depressive symptoms and suicide attempts: a systematic review of longitudinal studies. PLOS Med 2013; 10: e1001439.

10 Shamblaw AL, Cardy RE, Prost E, Harkness KL. Abuse as a risk factor for prenatal depressive symptoms: a meta-analysis. Arch Womens Ment Health 2019 22: $199-213$

11 Chmielowska M, Fuhr DC. Intimate partner violence and mental ill health among global populations of Indigenous women: a systematic review. Soc Psychiatry Psychiatr Epidemiol 2017; 52: 689-704.

12 Trevillion K, Oram S, Feder G, Howard LM. Experiences of domestic violence and mental disorders: a systematic review and meta-analysis. PLOS One 2012 7: e51740

13 Suglia SF, Duarte CS, Sandel MT. Housing quality, housing instability, and maternal mental health. J Urban Heal 2011; 88: 1105-16.

14 Pietrzak RH, Goldstein RB, Southwick SM, Grant BF. Prevalence and axis I comorbidity of full and partial posttraumatic stress disorder in the United States: results from wave 2 of the National Epidemiologic Survey on Alcoho and Related Conditions. J Anxiety Disord 2011; 25: 456-65.
15 Khalifeh $\mathrm{H}$, Oram S, Osborn D, Howard LM, Johnson S. Recent physical and sexual violence against adults with severe mental illness: a systematic review and meta-analysis. Int Rev Psychiatry 2016; 28: 433-51.

16 Blak BT, Thompson M, Dattani H, Bourke A. Generalisability of The Health Improvement Network (THIN) database: demographics, chronic disease prevalence and mortality rates. Inform Prim Care 2011; 19: 251-5.

17 NHS Digital. Read Codes. NHS Digital, 2017 (https://digital.nhs.uk/article/1104/ Read-Codes).

18 Booth N. What are the read codes? Health Libr Rev 1994; 11: 177-82.

19 Lévesque LE, Hanley JA, Kezouh A, Suissa S. Problem of immortal time bias in cohort studies: example using statins for preventing progression of diabetes. BMJ 2010; 340: b5087.

20 Townsend P, Phillimore P, Beattie A. Health and Deprivation: Inequality and the North. Croom Helm Google Scholar, 1988

21 Hegarty K. Domestic violence: the hidden epidemic associated with mental illness. Br J Psychiatry 2011; 198: 169-70.

22 Rodríguez M, Valentine JM, Son JB, Muhammad M. Intimate partner violence and barriers to mental health care for ethnically diverse populations of women. Trauma Violence Abuse 2009; 10: 358-74.

23 Feder G, Davies RA, Baird K, Dunne D, Eldridge S, Griffiths C, et al. Identification and Referral to Improve Safety (IRIS) of women experiencing domestic violence with a primary care training and support programme: a cluster randomised controlled trial. Lancet 2011; 378: 1788-95.

24 Usta J, Taleb R. Addressing domestic violence in primary care: what the physician needs to know. Libyan J Med 2014; 9: 23527.

25 Trevillion K, Hughes B, Feder G, Borschmann R, Oram S, Howard LM. Disclosure of domestic violence in mental health settings: a qualitative meta-synthesis. Int Rev Psychiatry 2014; 26: 430-44.

26 National Institute for Health and Care Excellence (NICE). Overview | Domestic Violence and Abuse: Multi-agency Working: Public Health Guideline 50 [PH50]. NICE, 2014 (https://www.nice.org.uk/guidance/ph50).

27 Stewart DE, Chandra PS. WPA international competency-based curriculum for mental health providers on intimate partner violence and sexual violence against women. World Psychiatry 2017; 16: 223-4.

28 World Psychiatric Association (WPA). WPA 2014-2017 Action Plan. WPA, 2014 (http://www.wpanet.org/detail.php?section_id=25\&content_id=1132).

29 Standing Together against Domestic Violence. Pathfinder. Standing Together against Domestic Violence, 2017 (http://www.standingtogether.org.uk/localpartnership/pathfinder).

30 Office for National Statistics. Intimate Personal Violence and Partner Abuse. Office for National Statistics, 2016 (https://www.ons.gov.uk/peoplepopulationandcommunity/crimeandjustice/compendium/focusonviolentcrimeandsexualoffences/yearendingmarch2015/ chapter4intimatepersonalviolenceandpartnerabuse).

31 Patel R, Shetty $\mathrm{H}$, Jackson $\mathrm{R}$, Broadbent $\mathrm{M}$, Stewart R, Boydell J, et al. Delays before diagnosis and initiation of treatment in patients presenting to mental health services with bipolar disorder. PLOS One 2015; 10: e0126530. 